\title{
ALMOST GLOBAL SOLUTIONS OF THE FREE BOUNDARY PROBLEM FOR THE EQUATIONS OF A MAGNETOHYDRODYNAMIC INCOMPRESSIBLE FLUID
}

Abstract. Almost global in time existence of solutions for equations describing the motion of a magnetohydrodynamic incompressible fluid in a domain bounded by a free surfaced is proved. In the exterior domain we have an electromagnetic field which is generated by some currents which are located on a fixed boundary. We prove that a solution exists for $t \in(0, T)$, where $T>0$ is large if the data are small.

1. Introduction. In this paper we prove the existence of almost global in time solutions for small data to equations describing the motion of a magnetohydrodynamic incompressible fluid in a domain $\Omega_{t} \subset \mathbb{R}^{3}$ bounded by a free surface $S_{t}$. In the domain $D_{t} \subset \mathbb{R}^{3}$ which is exterior to $\Omega_{t}$ we have a gas under constant pressure $p_{0}$. Moreover in the domain $D_{t}$ we have an electromagnetic field which is generated by some currents which are located on a fixed boundary $B$ on $D_{t}$.

In the domain $\Omega_{t}$ the motion is described by the following problem:

$$
\begin{array}{ll}
v_{t}+v \cdot \nabla v-\operatorname{div} \mathbb{T}(v, p)-\mu_{1} \stackrel{1}{H} \cdot \nabla \stackrel{1}{H}+\frac{1}{2} \mu_{1} \nabla \stackrel{1}{H^{2}}=f & \text { in } \widetilde{\Omega}^{T}, \\
\operatorname{div} v=0 & \text { in } \widetilde{\Omega}^{T}, \\
\mu_{1} \stackrel{1}{H}_{t}=-\operatorname{rot} \stackrel{1}{E} & \text { in } \widetilde{\Omega}^{T}, \\
\operatorname{rot} \stackrel{1}{H}=\sigma_{1}\left(\stackrel{1}{E}+\mu_{1} v \times \stackrel{1}{H}\right) & \text { in } \widetilde{\Omega}^{T}, \\
\operatorname{div}\left(\mu_{1} \stackrel{1}{H}\right)=0 & \text { in } \widetilde{\Omega}^{T} .
\end{array}
$$

2000 Mathematics Subject Classification: 35A05, 35R35, 76N10.

Key words and phrases: free boundary, local existence, Sobolev spaces, magnetohydrodynamic incompressible fluid.

Research partially supported by KBN grant no. 2PO3A00223. 
where $\widetilde{\Omega}^{T}=\bigcup_{0 \leq t \leq T} \Omega_{t} \times\{t\}, v=v(x, t)$ is the velocity of the fluid, $p=$ $p(x, t)$ is the pressure, $\stackrel{1}{H}=\stackrel{1}{H}(x, t)$ is the magnetic field, $f=f(x, t)$ is the external force field per unit mass, $\mu_{1}$ is the constant magnetic permeability, $\sigma_{1}$ is the constant electric conductivity, $\stackrel{1}{E}=\stackrel{1}{E}(x, t)$ is the electric field, and

$$
\mathbb{T}(v, p)=\left\{\nu\left(\partial_{x_{i}} v_{j}+\partial_{x_{j}} v_{i}\right)-p \delta_{i j}\right\}
$$

is the stress tensor, where $\nu$ is the viscosity of the fluid. Moreover, by

$$
\mathbb{D}(v)=\left\{\nu\left(\partial_{x_{i}} v_{j}+\partial_{x_{j}} v_{i}\right)\right\}
$$

we denote the dilatation tensor.

In the domain $D_{t}$ which is a dielectric (gas) we assume that there is no fluid motion inside $(v=0)$. Therefore we have only the electromagnetic field described by the following system:

$$
\begin{array}{ll}
\mu_{2} \stackrel{1}{H}_{t}=-\operatorname{rot} \stackrel{2}{E} & \text { in } \widetilde{D}^{T}, \\
\operatorname{rot} \stackrel{2}{H}=\sigma_{2} \stackrel{2}{E} & \text { in } \widetilde{D}^{T}, \\
\operatorname{div}\left(\mu_{2} \stackrel{2}{H}\right)=0 & \text { in } \widetilde{D}^{T},
\end{array}
$$

where $\widetilde{D}^{T}=\bigcup_{0 \leq t \leq T} D_{t} \times\{t\}$.

On $S_{t}=\partial \Omega_{t} \cap \partial D_{t}$ we assume the following transmission and boundary conditions:

$$
\begin{array}{ll}
n \mathbb{T}(v, p)=-p_{0} n & \text { on } \widetilde{S}^{T}, \\
\frac{1}{\sigma_{1}} \stackrel{1}{H}=\frac{1}{\sigma_{2}} \stackrel{2}{H} & \text { on } \widetilde{S}^{T}, \\
\stackrel{1}{E} \cdot \tau_{\alpha}=\stackrel{2}{E} \cdot \tau_{\alpha}, \quad \alpha=1,2, & \text { on } \widetilde{S}^{T}, \\
v n=-\frac{\phi_{t}}{\left|\nabla \phi_{t}\right|} & \text { on } \widetilde{S}^{T},
\end{array}
$$

where $\widetilde{S}^{T}=\bigcup_{0 \leq t \leq T} S_{t} \times\{t\}, n$ is the unit outward vector to $\Omega_{t}$ and normal to $S_{t}, \tau_{\alpha}, \alpha=1,2$, is any tangent vector to $S_{t}, \phi(x, t)=0$ describes $S_{t}$ at least locally.

Next we assume the boundary conditions on $B$ :

$$
\begin{array}{ll}
\stackrel{2}{H}=H_{*} & \text { on } B, \\
\stackrel{2}{E}=E_{*} & \text { on } B .
\end{array}
$$


Finally, we assume the initial conditions

$$
\begin{array}{lll}
\Omega_{t \mid t=0}=\Omega, & S_{t \mid t=0}=S, & D_{t \mid t=0}=D, \\
v_{\mid t=0}=v_{0}, & \stackrel{1}{H}_{\mid t=0}=\stackrel{1}{H}_{0} & \text { in } \Omega, \\
\stackrel{2}{H} & \text { in } D .
\end{array}
$$

To prove existence of solutions to the above problem we introduce the Lagrangian coordinates $\xi \in \Omega$. They are the initial data for the Cauchy problem

$$
\frac{d x}{d t}=v(x, t), \quad x_{\mid t=0}=\xi \in \Omega .
$$

Therefore $x_{v}(\xi, t)=\xi+\int_{0}^{t} \bar{v}(\xi, \tau) d \tau$, where

$$
\bar{v}(\xi, t)=v\left(x_{v}(\xi, t), t\right) .
$$

To introduce the Lagrangian coordinates in $D_{t}$ we extend $v$ onto $D_{t}$. Let us denote the extended function by $v^{\prime}$. Then we define $\xi \in D$ to be the Cauchy data for the problem

$$
\frac{d x}{d t}=v^{\prime}(x, t), \quad x_{\mid t=0}=\xi \in D .
$$

Therefore $x_{v^{\prime}}(\xi, t)=\xi+\int_{0}^{t} \bar{v}^{\prime}(\xi, \tau) d \tau$, where $\bar{v}^{\prime}(\xi, t)=v^{\prime}\left(x_{v^{\prime}}(\xi, t), t\right)$.

Then by $(1.1)_{5}$,

$$
\begin{aligned}
& \Omega_{t}=\left\{x \in \mathbb{R}^{3}: x=x_{v}(\xi, t), \xi \in \Omega\right\}, \\
& S_{t}=\left\{x \in \mathbb{R}^{3}: x=x_{v}(\xi, t), \xi \in S\right\} .
\end{aligned}
$$

Since $S_{t}$ is determined at least locally by the equation $\phi(x, t)=0, S$ is described by $\phi\left(x_{v}(\xi, t), t\right)_{\mid t=0}=0$. Moreover we have

$$
\bar{n}_{v}=n\left(x_{v}(\xi, t), t\right)=\left.\frac{\nabla_{x} \phi(x, t)}{\left|\nabla_{x} \phi(x, t)\right|}\right|_{x=x_{v}(\xi, t)} .
$$

To simplify considerations we introduce the following notation:

$$
\begin{aligned}
& \|u\|_{l, Q}=\|u\|_{H^{l}(Q)}, \quad Q \in\{\Omega, S, D, \Pi, B\}, 0 \leq l \in \mathbb{Z}, \\
& \|u\|_{k, p, q, Q^{T}}=\|u\|_{L_{q}\left(0, T, W_{p}^{k}(Q)\right)}, \quad Q \in\{\Omega, S, D, \Pi, B\}, \\
& p, q \in[1, \infty], \quad 0 \leq k \in \mathbb{Z},
\end{aligned}
$$

where $Q^{t}=Q \times(0, t)$,

$$
|u|_{p, Q}=\|u\|_{L_{p}(Q)}, \quad Q \in\{\Omega, S, D, \Pi, B\}, p \in[1, \infty] .
$$

2. Weak solutions. Weak solutions to problem (1.1)-(1.7) are defined in Lagrangian coordinates. 
Definition 2.1. By weak solutions to problem (1.1)-(1.7) we mean functions $\bar{v}, \bar{H}$ which satisfy the integral identities

$$
\begin{aligned}
\int_{0}^{T} \int_{\Omega}\left(-\bar{v} \bar{\varphi}_{t}+\mathbb{D}_{v}(\bar{v}) \mathbb{D}_{v}(\bar{\varphi})\right) d \xi d t & \\
& -\int_{0}^{T} \int_{\Omega}\left(\mu_{1} \frac{1}{H} \nabla_{v} \bar{H} \bar{\varphi}-\frac{1}{2} \mu_{1} \nabla_{v} \bar{H}^{2} \bar{\varphi}\right) d \xi d t \\
& =\int_{0}^{T} \int_{\Omega} \bar{f} \bar{\varphi} d \xi d t-\int_{0}^{T} \int_{S} p_{0} \bar{n}_{v} \varphi d \xi_{S} d t-\int_{\Omega} \bar{v}_{0} \bar{\varphi}(0) d \xi
\end{aligned}
$$

$$
\begin{aligned}
\int_{0}^{T} \int_{\Pi}\left(-\mu \bar{H} \bar{\psi}_{t}-\mu \bar{v} \nabla_{v} \bar{H} \bar{\psi}+\frac{1}{\sigma} \operatorname{rot}_{v} \bar{H} \operatorname{rot}_{v} \bar{\psi}\right) d \xi d t \\
-\int_{0}^{T} \int_{\Omega} \mu_{1}(\bar{v} \times \bar{H}) \operatorname{rot}_{v} \bar{\psi} d \xi d t \\
=\frac{1}{\sigma_{2}} \int_{0}^{T} \int_{B}\left(\bar{n}_{v} \times \bar{E}_{*}\right) \bar{\psi} d \xi_{B} d t-\mu \int_{\Pi} \bar{H}_{0} \bar{\psi}(0) d \xi
\end{aligned}
$$

where $\varphi, \psi$ are sufficiently regular with $\varphi(x, T)=\psi(x, T)=0$, and $\bar{n}_{v}$ is the unit outward vector normal to $S$ or $B$.

In $(2.1),(2.2)$ we use the notation $\bar{A} \xi, t)=A\left(x_{v}(\xi, t), t\right)$,

$$
\begin{aligned}
& \bar{H}_{\mid \Omega}=\stackrel{1}{H}, \quad \bar{H}_{\mid D}=\stackrel{2}{H}, \quad \sigma_{\mid \Omega}=\sigma_{1}, \quad \sigma_{\mid D}=\sigma_{2}, \quad \Pi=\Omega \cup D, \\
& \mu_{\mid \Omega}=\mu_{1}, \quad \mu_{\mid D}=\mu_{2},
\end{aligned}
$$

in $(2.2) v$ is extended over $\Pi$,

$$
\begin{aligned}
& \mathbb{D}_{v}(\bar{v})=\left\{\mu\left(\partial_{x_{i}} \xi_{k} \nabla_{\xi_{k}} \bar{v}_{j}+\partial_{x_{j}} \xi_{k} \nabla_{\xi_{k}} \bar{v}_{i}\right)\right\}, \quad \operatorname{rot}_{v} \bar{v}=\nabla_{v} \times \bar{v} \\
& \nabla_{v}=\partial_{x} \xi_{i} \nabla_{\xi_{i}}, \quad \operatorname{div}_{v} \bar{v}=\nabla_{v} \cdot \bar{v}=\partial_{x_{i}} \xi_{k} \nabla_{\xi_{k}} \bar{v}_{i}, \quad \partial_{\xi_{i}}=\nabla_{\xi_{i}} .
\end{aligned}
$$

Let $A$ be the Jacobi matrix of the transformation $x=x_{v}(\xi, t)$. Then $\operatorname{det} A=$ $\exp \left(\int_{0}^{t} \operatorname{div}_{v} \bar{v} d \tau\right)=1$. Moreover

$$
x_{\xi j}^{i}=\delta_{i j}+\int_{0}^{t} \partial_{\xi j} \bar{v}^{i}(\xi, \tau) d \tau, \quad \xi_{x}=x_{\xi}^{-1} .
$$

Hence we get

$$
\begin{aligned}
\sup _{\xi \in \Omega}\left|x_{\xi}\right| & \leq 1+\sup _{\xi \in \Omega} \int_{0}^{t}|\bar{v}(\xi, \tau)| d \tau \leq 1+c \int_{0}^{t}\|\bar{v}\|_{3, \Omega} d \tau \\
& \leq 1+c \sqrt{t}\left(\int_{0}^{t}\|\bar{v}\|_{3, \Omega}^{2} d \tau\right)^{1 / 2} \leq 1+c \sqrt{t}\|\bar{v}\|_{3,2,2, \Omega^{t}}
\end{aligned}
$$


and $\sup _{x \in \Omega_{t}}\left|\xi_{x}\right| \leq \varphi(a)$, where $a=\sqrt{t}\|\bar{v}\|_{3,2,2, \Omega^{t}}$ and $\varphi$ is an increasing positive function.

To prove the existence of a solution to the above problem we introduce Lagrangian coordinates connected with a given divergence-free function $u$. Moreover we linearize the terms with $v$ in (1.1) by writing them in the form $u \nabla v$ and $u \times \stackrel{1}{H}$. Then from $(2.1),(2.2)$ we get

$$
\begin{aligned}
& \int_{0}^{T} \int_{\Omega}\left(-\bar{v} \bar{\varphi}_{t}+\mathbb{D}_{u}(\bar{v}) \mathbb{D}_{u}(\bar{\varphi})\right) d \xi d t-\int_{0}^{T} \int_{\Omega}\left(\mu_{1} \bar{H}^{\prime} \nabla_{u} \bar{H}^{\prime} \bar{\varphi}\right. \\
& \left.-\mu_{1} \nabla_{u} \bar{H}^{\prime 2} \bar{\varphi}\right) d \xi d t=\int_{0}^{T} \int_{\Omega} \bar{f} \bar{\varphi} d \xi d t-\int_{0}^{T} \int_{S} p_{0} \bar{n}_{u} \varphi d \xi_{S} d t-\int_{\Omega} \bar{v}_{0} \bar{\varphi}(0) d \xi
\end{aligned}
$$

$$
\begin{gathered}
\int_{0}^{T} \int_{\Pi}\left(-\mu \bar{H} \bar{\psi}_{t}-\mu \bar{u} \nabla_{u} \bar{H} \bar{\psi}+\frac{1}{\sigma} \operatorname{rot}_{u} \bar{H} \operatorname{rot}_{u} \bar{\psi}\right) d \xi d t \\
-\int_{0}^{T} \int_{\Omega} \mu_{1}(\bar{u} \times \stackrel{1}{H}) \operatorname{rot}_{u} \bar{\psi} d \xi d t \\
=\frac{1}{\sigma_{2}} \int_{0}^{T} \int_{B}\left(\bar{n}_{u} \times \bar{E}_{*}\right) \bar{\psi} d \xi_{B} d t-\mu \int_{\Pi} \bar{H}_{0} \bar{\psi}(0) d \xi,
\end{gathered}
$$

where $\frac{1}{H^{\prime}}$ is a given function.

We write $(1.5)_{1}$ in the form $\bar{n} \mathbb{T}\left(\bar{v}, \bar{p}^{\prime}\right)=0$, where $\bar{p}=\bar{p}^{\prime}+p_{0}$. Then from Lemmas $3.1-3.9$ of [2] we get

$$
\begin{aligned}
& \left\|\bar{v}_{t}\right\|_{0, \Omega}^{2}+\|\bar{v}\|_{1, \Omega}^{2}+\|\bar{v}\|_{3,2,2, \Omega^{t}}^{2}+\left\|\bar{v}_{t}\right\|_{2,2,2, \Omega^{t}}^{2}+\left\|\bar{v}_{t t}\right\|_{1,2,2, \Omega^{t}}^{2} \\
& +\left\|\bar{v}_{t t}\right\|_{0, \Omega}^{2}+\left\|\bar{p}^{\prime}\right\|_{2,2,2, \Omega^{t}}^{2}+\left\|\bar{p}_{t}^{\prime}\right\|_{1,2,2, \Omega^{t}}^{2} \\
\leq & \alpha(a, t)\left[\left(t\left\|\bar{u}_{t}\right\|_{2,2,2, \Omega^{t}}^{2}+\|\bar{u}(0)\|_{0, \Omega}^{2}\right)\left(X_{1}+\bar{F}\right)+\left(\varepsilon\left(X_{1}+\bar{F}\right)\right.\right. \\
& \left.+c(\varepsilon) t\left(\bar{F}+\|\bar{v}(0)\|_{0, \Omega}^{2}\right)\right)\left\|\bar{u}_{t}\right\|_{1,2, \infty, \Omega^{t}}^{2} \\
& +\left(\varepsilon\left(\left\|\frac{1}{\bar{H}_{t}^{\prime}}\right\|_{2,2,2, \Omega^{t}}^{2}+\left\|\frac{1}{H^{\prime}}\right\|_{3,2,2, \Omega^{t}}^{2}\right)+c(\varepsilon) t\left(\left\|\bar{H}^{\prime}\right\|_{0,2,2, \Omega^{t}}^{2}+\left\|\bar{H}_{t t}^{\prime}\right\|_{0,2,2, \Omega^{t}}^{2}\right.\right. \\
& \left.\left.+\left\|\frac{1}{\bar{H}^{\prime}}(0)\right\|_{0, \Omega}^{2}\right)\right)\left(\left\|\bar{H}^{\prime}\right\|_{2,2, \infty, \Omega^{t}}^{2}\left(1+\|\bar{u}\|_{2,2, \infty, \Omega^{t}}^{2}\right)+\left\|\frac{1}{\bar{H}_{t}^{\prime}}\right\|_{2,2,2, \Omega^{t}}^{2}\right) \\
& +\left\|\bar{H}^{\prime}\right\|_{2,2, \infty, \Omega^{t}}^{2}\left(\varepsilon\left\|\bar{u}_{t}\right\|_{2,2,2, \Omega^{t}}^{2}+c(\varepsilon) t\left(\|\bar{u}\|_{0,2,2, \Omega^{t}}^{2}+\left\|\bar{u}_{t}(0)\right\|_{0, \Omega}^{2}\right)\right) \\
& \left.+\left\|\bar{f}_{t t}\right\|_{0,2,2, \Omega^{t}}^{2}+\left\|v_{t t}(0)\right\|_{0, \Omega}^{2}+\left\|\bar{f}_{t}\right\|_{0,2,2, \Omega^{t}}^{2}+\bar{X}_{1}+\bar{F}\right],
\end{aligned}
$$


where

$$
\begin{aligned}
\bar{F}= & \alpha_{1}(a, t)\left[\varepsilon\left\|\bar{H}^{\prime}\right\|_{3,2,2, \Omega^{t}}^{2}+c(\varepsilon) t\left(\left\|\bar{H}^{\prime}(0)\right\|_{0, \Omega}^{2}+\left\|\bar{H}_{t}^{\prime}\right\|_{0,2,2, \Omega^{t}}^{2}\right)\right) \\
& \cdot\left(\left\|\bar{H}^{\prime}\right\|_{0,2, \infty, \Omega^{t}}^{2}+\left\|\bar{H}^{\prime}\right\|_{2,2, \infty, \Omega^{t}}^{2}\right) \\
& +\left(\varepsilon\|\bar{u}\|_{3,2,2, \Omega^{t}}^{2}+c(\varepsilon) t\left(\left\|\bar{u}_{t}\right\|_{0,2,2, \Omega^{t}}^{2}+\|\bar{u}(0)\|_{0, \Omega}^{2}\right)\right)\left\|\bar{H}^{\prime}\right\|_{1,2, \infty, \Omega^{t}}^{4} \\
& \left.+c(\varepsilon)\left(\|\bar{f}\|_{0,2,2, \Omega^{t}}^{2}+\left\|\bar{f}_{t}\right\|_{0,2,2, \Omega^{t}}^{2}\right)+\|\bar{v}(0)\|_{1, \Omega}^{2}+\left\|\bar{v}_{t}(0)\right\|_{0, \Omega}^{2}\right],
\end{aligned}
$$

$$
\begin{aligned}
X_{1}=\alpha_{2}(a, t)[( & \left.\left\|\frac{1}{\bar{H}^{\prime}}\right\|_{3,2,2, \Omega^{t}}^{2}+\left\|\frac{1}{\bar{H}^{\prime}}\right\|_{2,2, \infty, \Omega^{t}}^{2}\right)\left(\varepsilon\left\|\frac{1}{\bar{H}^{\prime}}\right\|_{3,2,2, \Omega^{t}}^{2}\right. \\
& \left.\left.+c(\varepsilon)\left(\left\|\bar{H}^{\prime}(0)\right\|_{0, \Omega}^{2}+\left\|\bar{H}^{\prime}\right\|_{0,2,2, \Omega^{t}}^{2}\right)\right)+\|\bar{f}\|_{1,2,2, \Omega^{t}}^{2}\right] .
\end{aligned}
$$

From Lemmas 4.1-4.8 of [2] we get

$$
\left\|\bar{H}_{t}\right\|_{0, \Pi}^{2}+\|\bar{H}\|_{1, \Pi}^{2}+\|\bar{H}\|_{3,2,2, \Pi^{t}}^{2}+\left\|\bar{H}_{t}\right\|_{2,2,2, \Pi^{t}}^{2}
$$

$$
+\left\|\bar{H}_{t t}\right\|_{1,2,2, \Pi^{t}}^{2}+\left\|\bar{H}_{t t}\right\|_{0, \Pi}^{2}
$$

$$
\leq \alpha(a, t)\left[\left(\varepsilon\left(\bar{Y}_{1}+\left\|\bar{H}_{*}\right\|_{3,2,2, B^{t}}^{2}+\left\|\bar{u}_{t}\right\|_{2,2,2, \Pi^{t}}^{2}\right)\right.\right.
$$$$
+c(\varepsilon) t\left(\bar{G}+\bar{Y}_{2}+\left\|\bar{H}_{* t}\right\|_{0,2,2, B^{t}}^{2}+\left\|\bar{H}_{* t t}\right\|_{0,2,2, B^{t}}^{2}+\left\|\bar{u}_{t}\right\|_{0,2,2, \Pi^{t}}^{2}\right.
$$$$
\left.\left.+\left\|\bar{u}_{t t}\right\|_{0,2,2, \Pi^{t}}^{2}++\|\bar{u}(0)\|_{0, \Pi}^{2}+\left\|\bar{u}_{t}(0)\right\|_{0, \Pi}^{2}+\|\bar{H}(0)\|_{0, \Pi}^{2}+\left\|\bar{H}_{t}(0)\right\|_{0, \Pi}^{2}\right)\right)
$$$$
\cdot\left(\left\|\bar{u}_{t}\right\|_{2,2,2, \Pi^{t}}^{2}+\left\|\bar{u}_{t}\right\|_{1,2, \infty, \Pi^{t}}^{2}+\|\bar{u}(0)\|_{2, \Pi}^{2}+\|\bar{H}(0)\|_{2, \Pi}^{2}\right)+\varepsilon\left\|\bar{u}_{t}\right\|_{2,2,2 \Pi^{t}}^{4}
$$$$
+\left\|\bar{H}_{* t}\right\|_{1,2, \infty, B^{t}}^{2}+\left\|\bar{H}_{* t}\right\|_{1,2,2, B^{t}}^{4}+\left\|H_{* t}\right\|_{2,2,2, B^{t}}^{2}
$$$$
\left.+\left(t\left\|u_{t}\right\|_{2,2,2, \Pi^{t}}^{2}+\|\bar{u}(0)\|_{2, \Pi}^{2}\right) \bar{Y}_{1}+\bar{G}^{2}+\bar{G}\right],
$$

where

$$
\begin{aligned}
\bar{G}= & \alpha_{1}(a, t)\left[\left(\varepsilon\|\bar{u}\|_{3,2,2, \Pi^{t}}^{2}+c(\varepsilon) t\left(\left\|\bar{u}_{t}\right\|_{0,2,2, \Pi^{t}}^{2}+\|u(0)\|_{0, \Pi}^{2}\right)\right)\right. \\
& \left.\cdot\left\|\bar{E}_{*}\right\|_{1,2, \infty, B^{t}}^{2}+\|\bar{H}(0)\|_{1, \Pi}^{2}+\left\|\bar{H}_{t}(0)\right\|_{0, \Pi}^{2}+\left\|\bar{E}_{* t}\right\|_{0,2,2, B^{t}}^{2}\right] \\
\bar{Y}_{1}= & \alpha_{2}(a, t)\left[\left(\left(1+\|\bar{u}\|_{2,2, \infty, \Pi^{t}}^{2}+\|\bar{u}\|_{3,2,2, \Pi^{t}}^{2}\right.\right.\right. \\
& \left.+\|\bar{u}\|_{2,2, \infty, \Pi^{t}}^{4}+\left\|\bar{u}_{t}\right\|_{2,2,2, \Pi^{t}}^{2}\right) \\
& \cdot\left(\varepsilon\|\bar{u}\|_{3,2,2, \Pi^{t}}^{2}+c(\varepsilon) t\left(\left\|\bar{u}_{t}\right\|_{0,2,2, \Pi^{t}}^{2}\right.\right. \\
& \left.\left.+\|\bar{u}(0)\|_{0, \Pi}^{2}\right)\right)\left(\|\bar{u}\|_{2,2, \infty, \Pi^{t}}^{2}+1\right) \\
& \left.+\|\bar{H}(0)\|_{0, \Pi}^{2}+\left\|\bar{H}_{*}\right\|_{3,2,2, B^{t}}^{2}+\left\|\bar{H}_{*}(0)\right\|_{0, B}^{2}\right)\left(t\left\|\bar{u}_{t}\right\|_{2,2,2, \Pi}^{2}\right. \\
& \left.+\|\bar{u}(0)\|_{2, \Pi}^{2}+1\right)^{2}+t\left\|\bar{u}_{t}\right\|_{2,2,2, \Pi^{t}}^{2}+\|\bar{u}(0)\|_{2, \Pi}^{2}+\varepsilon \bar{u}_{t} \|_{2,2,2, \Pi^{t}}^{2} \\
& +c(\varepsilon) t\left(\left\|\bar{u}_{t t}\right\|_{0,2,2, \Pi^{t}}^{2}+\left\|\bar{u}_{t}(0)\right\|_{0, \Pi}^{2}\right)+\left\|\bar{H}_{* t}\right\|_{2,2,2, B^{t}}^{2} \\
& \left.+\left\|\bar{H}_{* t t}\right\|_{0,2,2, B^{t}}^{2}+\|\bar{H}(0)\|_{1, \Pi}\right]
\end{aligned}
$$




$$
\begin{aligned}
\bar{Y}_{2}= & \alpha_{3}(a, t)\left[\varepsilon \left(\left\|\bar{u}_{t t}\right\|_{1,2,2, \Pi^{t}}^{4}+\left\|\bar{u}_{t}\right\|_{1,2, \infty, \Pi^{t}}^{4}\|\bar{u}\|_{2,2, \infty, \Pi^{t}}^{4}\right.\right. \\
& \left.+\left\|\bar{u}_{t}\right\|_{1,2, \infty, \Pi^{t}}^{4}+\|\bar{u}\|_{2,2, \infty, \Pi^{t}}^{4}\left\|\bar{u}_{t t}\right\|_{1,2,2, \Pi^{t}}^{4}\right)\left(t^{2} \bar{G}^{2}+\|\bar{H}(0)\|_{1, \Pi}^{4}\right) \\
& +\left(\varepsilon\|\bar{u}\|_{3,2,2, \Pi^{t}}^{2}+c(\varepsilon) t\left(\|\bar{u}\|_{0,2,2, \Pi^{t}}^{2}\right.\right. \\
& \left.\left.+\left\|\bar{u}_{t}(0)\right\|_{0, \Pi}^{2}\right)\right)\left(\left\|\bar{u}_{t}\right\|_{1,2, \infty, \Pi^{t}}^{2}+1\right) \\
& \cdot\left\|\bar{H}_{t t}(0)\right\|_{0, \Pi}^{2}+\left\|\bar{u}_{t}\right\|_{1,2, \infty, \Pi^{t}}^{2}\left(\varepsilon \bar{Y}_{1}+c(\varepsilon) t\left(\bar{G}+\|\bar{H}(0)\|_{0, \Pi}^{2}\right)\right) \\
& \left.+\left\|\bar{E}_{*}\right\|_{0,2,2, B^{t}}^{2}+\left\|\bar{E}_{* t t}\right\|_{0,2,2, B^{t}}^{2}\right],
\end{aligned}
$$

where $\alpha_{i}, i=1,2,3$, are increasing positive functions.

\section{Existence of solutions to (1.1)-(1.7)}

Lemma 3.1. Let $\varepsilon=\sqrt{t}$ and $\bar{u}=\bar{v}_{m}, \bar{H}^{\prime}=\bar{H}_{m}$. Then from (2.5)-(2.11) we get

(3.1) $\quad \beta_{m+1}(t)$

$$
\begin{aligned}
\leq & \sum_{i=2}^{6} \alpha_{i}\left(t^{\gamma} B, t^{\gamma} \varphi(0), B, \varphi(0)\right) \beta_{m}^{i}(t)+c t^{\gamma}(B+\varphi(0)) \beta_{m}(t) \\
& +\alpha_{1}\left(t^{\gamma} B, t^{\gamma} \varphi(0), B, \varphi(0)\right)
\end{aligned}
$$

where

$$
\begin{aligned}
B= & \left\|\bar{E}_{*}\right\|_{0,2,2, B^{t}}+\left\|\bar{E}_{* t}\right\|_{0,2,2, B^{t}}^{2}+\left\|\bar{H}_{*}\right\|_{3,2,2, B^{t}}^{2}+\left\|\bar{H}_{* t}\right\|_{2,2,2, B^{t}}^{2} \\
& +\left\|\bar{E}_{* t t}\right\|_{0,2,2, B^{t}}^{2}+\left\|\bar{E}_{*}\right\|_{1,2, \infty, B^{t}}^{2}+\left\|\bar{H}_{* t t}\right\|_{0,2,2, B^{t}}^{2}+\left\|\bar{f}_{t}\right\|_{0,2,2, \Omega^{t}}^{2} \\
& +\|\bar{f}\|_{1,2,2, \Omega^{t}}^{2}+\left\|\bar{f}_{t t}\right\|_{0,2,2, \Omega^{t}}^{2} \\
\varphi(0)= & \sum_{i+k \leq 2}\left(\left\|\partial_{t}^{i} \bar{v}(0)\right\|_{k, \Omega}^{2}+\left\|\partial_{t}^{i} \bar{H}(0)\right\|_{k, \Pi}^{2}\right), \\
\beta_{m}(t)= & \left\|\bar{v}_{m t}\right\|_{0, \Omega}^{2}+\left\|\bar{v}_{m}\right\|_{1, \Omega}^{2}+\left\|\bar{v}_{m}\right\|_{3,2,2, \Omega^{t}}^{2}+\left\|\bar{v}_{m t}\right\|_{2,2,2, \Omega^{t}}^{2} \\
& +\left\|\bar{v}_{m t t}\right\|_{1,2,2, \Omega^{t}}^{2}+\left\|\bar{v}_{m t t}\right\|_{0, \Omega}^{2}+\left\|\bar{p}_{m}^{\prime}\right\|_{2,2,2, \Omega^{t}}^{2}+\left\|\bar{p}_{t m}^{\prime}\right\|_{1,2,2, \Omega^{t}}^{2} \\
& +\left\|\bar{H}_{m t}\right\|_{0, \Pi}^{2}+\left\|\bar{H}_{m}\right\|_{1, \Pi}^{2}+\left\|\bar{H}_{m}\right\|_{3,2,2, \Pi^{t}}^{2} \\
& +\left\|\bar{H}_{m t}\right\|_{2,2,2, \Pi^{t}}^{2}+\left\|\bar{H}_{m t t}\right\|_{1,2,2, \Pi^{t}}^{2}
\end{aligned}
$$

and $\alpha_{i}, i=1, \ldots, 6$, are polynomial functions $\left(\alpha_{1}(0,0,0,0)=0\right)$, and $\gamma>0$, $c>0$ are some constants.

REMARK. In (2.5)-(2.11) we use the inequalities

$$
\begin{aligned}
& \|\bar{u}\|_{2,2, \infty, \Omega^{t}}^{2} \leq c t\left(\left\|\bar{u}_{t}\right\|_{2,2,2, \Omega^{t}}^{2}+\|\bar{u}(0)\|_{2, \Omega}^{2}\right), \\
& \left\|\bar{H}^{\prime}\right\|_{2,2, \infty, \Pi^{t}}^{2} \leq c t\left(\left\|\bar{H}_{t}^{\prime}\right\|_{2,2,2, \Pi^{t}}^{2}+\left\|\bar{H}^{\prime}(0)\right\|_{2, \Pi}^{2}\right) .
\end{aligned}
$$


Lemma 3.2. Let $A>0$ be sufficiently small and let $B, \varphi(0)$ be such that

$$
\begin{aligned}
& \alpha_{1}\left(t^{\gamma} B, t^{\gamma} \varphi(0), B, \varphi(0)\right) \leq \frac{1}{3} A, \\
& c t^{\gamma}(B+\varphi(0)) \leq \frac{1}{3}, \\
& \sum_{i=2}^{6} \alpha_{i}\left(t^{\gamma} B, t^{\gamma} \varphi(0), B, \varphi(0)\right) A^{i-1} \leq \frac{1}{3} .
\end{aligned}
$$

Then $\beta_{m}(t) \leq A, m=1,2, \ldots$

Proof. Assume that $\beta_{m}(t) \leq A$. Then (3.1), (3.3) imply that $\beta_{m+1}(t) \leq A$.

REMARK. From (3.3) we obtain the qualitative formula

$$
t^{\gamma} \sim \frac{A}{B+\varphi(0)} .
$$

Then for given $A$ and small $B, \varphi(0)$ we can choose $t$ large.

From Lemma 5.2 of $[2]$ we get

$$
\begin{gathered}
\left\|\overline{\mathcal{V}}_{m+1}\right\|_{0, \Pi}^{2}+\left\|\overline{\mathcal{V}}_{m+1}\right\|_{2,2,2, \Pi^{t}}^{2}+\left\|\overline{\mathcal{V}}_{m+1 t}\right\|_{0,2,2, \Pi^{t}}^{2}+\left\|\overline{\mathcal{V}}_{m+1}\right\|_{1, \Pi}^{2} \\
+\left\|\overline{\mathcal{P}}_{m+1}^{\prime}\right\|_{1,2,2, \Omega^{t}}^{2}+\left\|\overline{\mathcal{H}}_{m+1}\right\|_{0, \Pi}^{2}+\left\|\overline{\mathcal{H}}_{m+1}\right\|_{1,2,2, \Pi^{t}}^{2} \\
\leq \alpha(A) t(t+1)\left(\left\|\overline{\mathcal{V}}_{m}\right\|_{2,2,2, \Pi^{t}}^{2}+\left\|\overline{\mathcal{H}}_{m}\right\|_{1,2,2, \Pi^{t}}^{2}\right),
\end{gathered}
$$

where $\overline{\mathcal{V}}_{m+1}=\bar{v}_{m+1}-\bar{v}_{m}, \overline{\mathcal{P}}_{m+1}^{\prime}=\bar{p}_{m+1}^{\prime}-\bar{p}_{m}^{\prime}, \overline{\mathcal{H}}_{m+1}=\bar{H}_{m+1}-\bar{H}_{m}$ and $\alpha(0)=0$.

Lemma 3.3. Let the assumptions of Lemma 3.2 be satisfied. Then for $t \leq T^{*}$, where $\alpha(A) T^{*}\left(T^{*}+1\right)<1$, we have convergence of the sequence $\left(\bar{v}_{m}, \bar{p}_{m}^{\prime}, \bar{H}_{m}\right)$.

THeOREM 3.1. Let the assumptions of Lemmas 3.1,3.2 be satisfied. Then for $T \leq T^{*}$ there exists a solution to problem (1.1)-(1.7) such that

$$
\begin{aligned}
& \bar{v} \in L_{2}\left(0, T, H^{3}(\Omega)\right) \cap L_{\infty}\left(0, T, H^{1}(\Omega)\right), \\
& \bar{v}_{t} \in L_{\infty}\left(0, T, H^{1}(\Omega)\right) \cap L_{2}\left(0, T, H^{2}(\Omega)\right), \\
& \bar{v}_{t t} \in L_{2}\left(0, T, H^{1}(\Omega)\right) \cap L_{\infty}\left(0, T, L_{2}(\Omega)\right), \\
& \bar{p}^{\prime} \in L_{2}\left(0, T, H^{2}(\Omega)\right), \\
& \bar{p}_{t}^{\prime} \in L_{2}\left(0, T, H^{1}(\Omega)\right), \\
& \bar{H} \in L_{2}\left(0, T, H^{3}(\Pi)\right) \cap L_{\infty}\left(0, T, H^{1}(\Pi)\right), \\
& \bar{H}_{t} \in L_{\infty}\left(0, T, H^{1}(\Pi)\right) \cap L_{2}\left(0, T, H^{2}(\Pi)\right), \\
& \bar{H}_{t t} \in L_{2}\left(0, T, H^{1}(\Pi)\right) \cap L_{\infty}\left(0, T, L_{2}(\Pi)\right)
\end{aligned}
$$


and

$$
\begin{aligned}
\left\|\bar{v}_{t}\right\|_{1,2, \infty, \Omega^{T}}^{2}+\|\bar{v}\|_{1,2, \infty, \Omega^{T}}^{2}+\|\bar{v}\|_{3,2,2, \Omega^{T}}^{2}+\left\|\bar{v}_{t}\right\|_{2,2,2, \Omega^{T}}^{2}+\left\|\bar{v}_{t t}\right\|_{1,2,2, \Omega^{T}}^{2} \\
+\left\|\bar{v}_{t t}\right\|_{0,2, \infty, \Omega^{T}}^{2}+\left\|\bar{p}^{\prime}\right\|_{2,2,2, \Omega^{T}}^{2}+\left\|\bar{p}_{t}^{\prime}\right\|_{1,2,2, \Omega^{T}}^{2}+\left\|\bar{H}_{t}\right\|_{1,2, \infty, \Pi^{T}}^{2} \\
+\|\bar{H}\|_{1,2, \infty, \Pi^{T}}^{2}+\|\bar{H}\|_{3,2,2, \Pi^{T}}^{2}+\left\|\bar{H}_{t}\right\|_{2,2,2, \Pi^{T}}^{2}+\left\|\bar{H}_{t t}\right\|_{0,2, \infty, \Pi^{T}}^{2} \\
\quad+\left\|\bar{H}_{t t}\right\|_{1,2,2, \Pi^{T}}^{2} \leq A .
\end{aligned}
$$

Acknowledgments. The author thanks Prof. W. Zajączkowski for very fruitful discussions during the preparation of this paper.

\section{References}

[1] O. V. Besov, V. P. Il'in, and S. M. Nikol'skiü, Integral Representations of Functions and Imbedding Theorems, Nauka, Moscow, 1975 (in Russian).

[2] P. Kacprzyk, Local existence of solutions of the free boundary problem for the equations of a magnetohydrodynamic incompressible fluid, Appl. Math. (Warsaw) 30 (2003), 461-488.

[3] L. Landau and E. Lifshits, Electrodynamics of Continuous Media, Nauka, Moscow, 1957 (in Russian).

[4] W. M. Zajączkowski, Existence of solutions for free boundary problems for viscous compressible barotropic fluids, Ann. Polon. Math. 60 (1995), 255-287.

Institute of Mathematics and Cryptology

Cybernetics Faculty

Military University of Technology

S. Kaliskiego 2

00-908 Warszawa, Poland

E-mail: p.kacprzyk@imbo.wat.edu.pl

Received on 17.6.2003;

revised version on 5.11.2003 